\title{
Prioritisation in healthcare—still muddling through
}

\author{
Bert Gordijn · Henk ten Have
}

Published online: 31 March 2011

(C) Springer Science+Business Media B.V. 2011

Would it not be wonderful, if we could always provide everybody with all the medical interventions they might require or fancy? Not in this world-neither in developing nor in developed nations. We are witnessing speedily mounting costs of emerging medical technologies and novel drugs. At the same time changing demographics imply that more and more elderly with a plethora of chronic diseases will have to be taken care of. However, resources for health care are not boundless. As national budgets are limited, channelling more money to health care wears away resources from other portfolios essential for health (e.g. schooling, education, housing and infrastructure), which at a certain stage starts having negative effects on public health.

We have seen unequalled advances in health sciences and medical technologies over the last decades, triggering equally unparalleled demands for new medical services, effective preventive measures, successful therapies and higher overall quality. In their new book Overdiagnosed: Making People Sick in the Pursuit of Health Welch et al. (2011) describe how medical diagnostics have advancedespecially medical imaging and genetic testing technologies- to the extent that hardly anything seems to be able to escape medical scrutiny today. As a result many pathology thresholds have shifted downward so as to include more and more patients needing treatment (e.g. blood pressure, cholesterol and osteoporosis). In addition, new labels such

B. Gordijn ( $\square)$

Institute of Ethics, Dublin City University, Dublin 9, Ireland

e-mail: bert.gordijn@dcu.ie

H. ten Have

Center for Healthcare Ethics, Duquesne University,

Pittsburgh, PA 15282, USA

e-mail: tenhaveh@duq.edu as predisposition, pre-diabetes and pre-obesity abound. People lacking any clinical symptoms but labeled as having pre-diseases are now seeking medical treatments (Welch et al. 2011).

Why only target patients and pre-patients as potential customers though? After all, you might as well attempt to sell your products to practically everybody. At the moment, this seems exactly what is happening. There is a surge of so-called 'enhancement drugs' and 'enhancement technologies' tapping into a potentially huge market. Of course, you must be able to convince perfectly normal people that they are not smart enough. Nor are they adequately goodlooking and happy. In actual fact, they could be more attractive, fitter and work longer hours and with much more focus, if they would only buy your smart drugs and happy pills. Obviously the financial interests for the pharmaceutical industry are enormous.

Against this backdrop it is increasingly important to think about ways of setting priorities in health care. Although there are advocates of forms of implicit prioritization (i.e. without trying to advance any criteria or principles), calls for greater clarity and accountability in health policy have triggered the establishment of explicit prioritization bodies in a variety of countries. Consequently, in the last few decades we have gained quite some experience with the selection of health care services for public funding. What are the results of these attempts at explicit prioritization?

In their review of eight different national experiences with priorities setting since the late 1980s Sabik and Lie (2008) argue that little progress has been made. They distinguish two different priority setting approaches: outlining principles as well as defining practices. Because of their abstractness, government commissions that have outlined principles (Netherlands, Norway, Sweden and 
Denmark) have had little direct impact on their countries' policies. In contrast, explicit priority setting efforts focused on concrete allocation decisions (Israel, New Zealand, the UK and the state of Oregon in the US) affected policy and practice more directly. Thus the authors argue that in order to ensure impact on policy and practice, it is imperative to establish bodies with some decision making power on what is actually implemented in the health care system. In addition, public engagement with prioritization issues is widely regarded as necessary to gain acceptance for the choices to be made as well as to ensure their fairness and legitimacy from a procedural point of view. However, the priorities setting exercises have hardly achieved a structural, transparent and participatory public involvement in decision making (Sabik and Lie 2008).

Likewise Kenny and Joffres (2008) are rather critical in their assessment of the international approaches to explicit prioritisation at the macro level. Their analysis focuses on attempts in six different countries (Norway, Netherlands, Sweden, Denmark, New Zealand, and the state of Oregon in the US). The authors argue that these prioritisation efforts have only resulted in insignificant changes in the allocation of resources based on priorities. Nor have they resulted in clear lists or packages of publicly funded core services. They attribute the failure to a deficiency in clarity and rigor regarding the ethical issues involved in explicit prioritisation as well as the lack of political will to put explicit priority setting into effect (Kenny and Joffres 2008).

The first paper in the current issue of Medicine, Health Care and Philosophy, 'Evidence, Ethics and Inclusion: a Broader Base for NICE', by Stephen Wilmot, contributes to the discussion about setting priorities and defining core services. Wilmot focuses on the National Institute for
Health and Clinical Excellence (NICE), which has been created to give assistance on which treatments should be provided out of taxes by the British National Health Service. He argues that NICE has not been able to adequately explain and justify its decisions in the public arena in terms of distributive justice. This has not only weakened NICE's legitimacy. It has affected the National Health Service as a whole. Also Wilmot develops an explanation as to whence NICE's failure arises. The Institute's over-reliance on positivist epistemology and its focus on liberal ethics limit its ability to communicate effectively with the community. In addition, he goes on to point out how this failure could be corrected by giving wider representation to the diversity of perspectives in contemporary western society (Wilmot 2011).

As it is increasingly urgent to adequately tackle the issue of setting priorities and defining core services, the editors welcome all attempts to advance the debate. At the moment, however, we very much seem to be still muddling through.

\section{References}

Kenny, N., and C. Joffres. 2008. An ethical analysis of international health priority-setting. Health Care Analysis 16(2): 145-160.

Sabik, L.M., and R.K. Lie. 2008. Priority setting in health care: Lessons from the Experiences of Eight Countries. International Journal for Equity in Health 7(1): 4. doi:10.1186/1475-9276-7-4.

Welch, H.G., L.M. Schwartz, and S. Woloshin. 2011. Overdiagnosed: Making People Sick in the Pursuit of Health. Boston: Beacon Press.

Wilmot, S. 2011. Evidence, ethics and inclusion: A broader base for NICE. Medicine, Health Care and Philosophy 14(2) this issue. 